national service. The cost of long distance calls is likely to be the main reason for this difference, and might be overcome by a freephone service should funding allow this.

The findings show that people require information in two key areas: medical and support services. The need for information about the disease and its treatment justifies the choice of oncology nurses to staff the service and in turn the provision of the medical and specialist advisory board.

As regards cancer site the predominance of inquiries about breast cancer is striking. This is the most common site discussed by men as well as women. Similarly, the number of calls about cervical and ovarian cancers are disproportionately high. A possible influence may be that these conditions feature prominently in womens' magazines and have also been the subject of recent controversy over screening and options for treatment. Moreover, it is reasonable to suppose, that the great interest in these cancers is related to their prevalence in younger patients and to issues about sexuality.

Although BACUP was envisaged predominantly as a telephone service, letters make up $30 \%$ of the inquiries. The more positive effect on mood reported by telephone inquirers in the user survey, however, suggests that a telephone service may be of particular value. While it is possible that those who returned their questionnaires were more likely to be happy with the service, the user survey produced an overwhelmingly positive response.

BACUP has been in operation for over two years and is now handling an average of 85 inquiries a day. The direct relation between publicity and the number of inquiries received suggests that use will increase as BACUP becomes more widely known.

The establishment of a national cancer information service was the inspiration of a doctor who discovered what it means to become a cancer patient. Vicky Clement-Jones recognised the need for an organisation which, working in conjunction with the medical and nursing professions, would improve the quality of information and emotional support received by cancer patients and their families. The results in this paper bear out the accuracy of this perception.

We thank the nurses of BACUP cancer information service: Anne Allbright, Sue Chambers, Nora Elwood, Mary Finn, Elaine Fleck, Linda Huby, Celia Key, Cecile Messent, Wendy Murnaghan, Sally Openshaw, Hilary Plant, Leslie Ridley, Diane Rumbelow, Fiona Scott, Mary Venn, Kevina Warner, and Nigel Williams; Walter Gregory for designing a statistical package tailored to our needs; Sheila Jones for her help in designing the user survey; and Theresa Shrimpton and Tracey Holliday for their help in compiling this report.

This project was funded by the Cancer Research Campaign.

1 Calman K, Calman A. Public response to cancer education in the press. Health Education fournal 1983;42:53-4.

2 Freimuth S, Stein JA, Kear TJ. Searching for health information-the cancer information service model. Philadelphia: University of Pennsylvania Press (in press)

3 Rayner C. The role of women's magazines in communication and cancer education. In: Deeley TJ, ed. Communication and cancer education. Cardiff: Alpha Omega, 1981:35-8. (Proceedings of a symposium organised by Tenovis Cancer Information Centre, March 1980.)

4 Calman K. Cancer line: an experiment in communication. Health Education foumal 1984;42:102-3.

5 Clement-Jones V. Cancer and beyond: the formation of BACUP. Br Med $\mathrm{J}$ 1985;291:1021-3.

6 Office of Population Censuses and Surveys. Cancer statistics registration, 1983. London: HMSO, 1986.

7 Office of Population Censuses and Surveys. Census, 1981. Economic activity, Great Britain. London: HMSO, 1984.

8 Griffin T, ed. Social trends No 17. London: HMSO, 1987.

9 Office of Population Censuses and Surveys. Population trends, 48. London: HMSO, 1987

10 Office of Population Censuses and Surveys. Population and vital statistics, local and health authority area, summary, 1984. London: HMSO, 1986.

11 Cassileth BP, Zupkis RV, Sutton-Smith K, March V. Information and participation preferences among cancer patients. Ann Intern Med 1980;92 $832-6$.

12 Townsend $\mathrm{P}$, Davidson $\mathrm{N}$, eds. Inequalities in health. Harmondsworth: Penguin, 1982. (Black report.)

13 Office of Population Censuses and Surveys. Labour force survey, 1983-1984. London: HMSO, 1986.

(Accepted 4 fuly 1988)

\title{
Graphical aid for determining power of clinical trials involving two groups
}

\author{
Douglas K Miller, Sharon M Homan
}

Department of Medicine, Saint Louis University School of Medicine, St Louis, Missouri, USA 63104

Douglas K Miller, $M D$, assistant professor of medicine

Center for Health Services Education and Research, St Louis University Medical

Center, St Louis, Missouri, USA 63104

Sharon M Homan, PHD, associate professor, Center for Health Services Education and Research

Correspondence and reprint requests to: Dr D K Miller, Division of Geriatrics, 1402 South Grand Boulevard, St Louis, MO 63104.

\begin{abstract}
Physicians need to evaluate clinical research critically, and determining the power of a study is an essential component of research evaluation. This report presents a graphical aid that permits rapid power determination for clinical trials with two groups. Power curves were developed for dichotomous outcomes by setting two tail $\alpha$ at 0.05 and varying the sample size, the control group response rate, and the clinically important difference between control and experimental groups as defined by the user. Use of the graphical aid was demonstrated to a group of 18 medical students, residents, fellows, and faculty in a 15 minute session. Evaluation of the trainees' application of the aid showed a small average bias of -0.0003 and an average variance of 0.006. Ninety percent of power estimates were within 0.05 of the true value determined by formula.

This graphical aid is recommended as a rapid and accurate method for determining power in the critical appraisal of clinical research.
\end{abstract}

\section{Introduction}

Physicians need to evaluate clinical research critically to keep up to date with current work' and to make sound decisions in patient management. ${ }^{2}$ Critical research evaluation requires an understanding of and ability to use the important components of biostatistical inference. ' A major issue in evaluating clinical trials is whether the research design has adequate power to show a statistically significant difference between study groups if in truth a clinically important difference exists. The graphical aid presented here is recommended as a rapid and simple method for estimating power. It can be used for evaluating published reports and for teaching the concepts of statistical power to medical trainees.

Many clinicians and researchers are uncertain how to interpret a negative clinical trial. For example, the Danish double blind controlled trial failed to find aspirin effective in reducing the incidence of transient ischaemic attacks or death. ${ }^{3}$ Dyken, however, questioned, "Does the failure of the Danish study to show any favourable effect mean that the other studies [showing positive effect of aspirin] are wrong?"' To answer such questions power analysis can be used to determine the probability that the trial will find a statistically significant difference when a specified difference really exists. To show how power analysis is used the concepts of type I and type II error in statistical significance testing are briefly reviewed. 
The aim of the two group randomised trial using dichotomous outcomes is to compare the response rates of the control and experimental groups. A type I error results when it is falsely concluded that there is a difference between the groups. The $\alpha$ value represents the pre-established acceptable probability of type I error. The $P$ value in published reports represents the likelihood of observing a difference between groups as large as the observed difference given the null hypothesis is true.

A type II error denotes the mistake of concluding that there is no difference between groups when a clinically important difference does exist, and the probability of its occurring is represented by $\beta$. The power of a trial is the arithmetic complement of $\beta, 1-\beta$, and represents the probability that a statistically significant difference will be found when a clinically important difference truly exists. Power is similar to the sensitivity of a diagnostic test in individual patient care. ${ }^{5}$ Thus, the possibility that a disease is present cannot be excluded by a negative result from an insensitive diagnostic test, and the possibility that a clinically important difference between research groups exists cannot be rejected by a negative clinical trial with low power.

The issues of the type II error rate and its complement, power, have been increasingly evident in published work. ${ }^{6.10}$ Several reports have shown that many clinical studies include too few patients to be able to detect clinically important outcomes. ${ }^{67}$ Ideally, the power of a trial should be reported as part of the study design; however, this is frequently not done..$^{6910}$

Several methods have been proposed for determining the proper sample size so that the null hypothesis will be rejected at a specified level of $\alpha$ if a clinically important difference between groups does exist. ${ }^{811-13}$ Recently Young et al presented easy to use, pocket sized nomograms for estimating the sample size required to achieve a power of $80 \%$ assuming a specified clinically important difference truly exists. ${ }^{12}$ Detsky and Sackett developed tables that consider the research findings when estimating the required sample size to avoid a false conclusion of equivalency when a $25 \%$ or $50 \%$ improvement in adverse outcomes really exists. ${ }^{13}$ The goal of our study, however, was not to assess the sample size needed to achieve a desired power (or its " $\alpha$ " problem analogue $e^{13}$ ) but rather to estimate the power ("sensitivity") of a clinical trial given the sample sizes actually used. Power can be accurately determined via available formulas, tables, ${ }^{81+16}$ or computer programs. These methods are, however, complicated and time consuming.

We developed a graphical aid that $(a)$ educators can use to teach trainees about power and $(b)$ clinicians can use in evaluating published research. To keep the instrument small and usable we tailored it to fit the most common clinical situations (as indicated by a review of the series of clinical trials reported by Freiman et $a l^{\dagger}$ ).

\section{Methods}

DEVELOPMENT OF THE GRAPHICAL AID

The following formula ${ }^{1+16}$ was used to construct the power graphs that constitute the aid:

$$
Z_{\text {power }}=\frac{Z_{\omega / 2} \sqrt{2 \overline{\mathrm{pq}}}-\left|\mathrm{p}_{\mathrm{E}}-\mathrm{p}_{\mathrm{C}}\right| \sqrt{\mathrm{n}-\left|\overline{\mathrm{p}_{\mathrm{E}}-\mathrm{p}_{\mathrm{C}}}\right|}}{\sqrt{\mathrm{p}_{\mathrm{C}} \mathrm{q}_{\mathrm{C}}+\mathrm{p}_{\mathrm{E}} \mathrm{q}_{\mathrm{E}}}}
$$

where $Z_{\text {power }}$ is the standard normal deviate corresponding to power; $Z_{u 2}$ is the normal curve value for two tailed test at $\alpha$ significance level; $\mathrm{p}_{\mathrm{C}}$ is the hypothesised proportion of respondents in the control group of size $n$; $\mathrm{p}_{\mathrm{E}}$ is the hypothesised proportion of respondents in the experimental or comparison group of size $n ; \bar{p}=\left(p_{E}+\right.$
$\left.\mathrm{p}_{\mathrm{C}}\right) / 2 ; \mathrm{q}_{\mathrm{C}}=\left(1-\mathrm{p}_{\mathrm{C}}\right) ; \mathrm{q}_{\mathrm{E}}=\left(1-\mathrm{p}_{\mathrm{E}}\right) ;$ and $\overline{\mathrm{q}}=(1-\overline{\mathrm{p}}) ; \mid \mathrm{p}_{\mathrm{E}}-$ $\mathrm{p}_{\mathrm{C}}$ defines the clinically important differences (not the observed difference). Use of this formula for estimating the statistical power of a two group comparison assumes that the two sample sizes are equal to a common $n$, that the samples are random and not matched, and that multiple testing is not performed.

As the formula shows, the power of the statistical test is a function of the preassigned significance level, $\alpha$; the sample size, $\mathrm{n}$; the hypothesised magnitude of $\mathrm{p}_{\mathrm{C}}$; and the absolute difference in response rates that it is clinically important to detect, $\left|\mathrm{p}_{\mathrm{E}}-\mathrm{p}_{\mathrm{C}}\right|$. Having established $\mathrm{p}_{\mathrm{C}}$ the user specifies the clinically important difference-that is, $\left|\mathrm{p}_{\mathrm{E}}-\mathrm{p}_{\mathrm{C}}\right|$ - and derives a hypothesised value for $\mathrm{p}_{\mathrm{E}}$. To construct graphical aid we set $\alpha$ at 0.05 (suitable for a two tailed test at $\alpha=0.05$ or a one tailed test at $\alpha=0 \cdot 1$ ), and determined power for multiple levels of $\mathrm{p}_{\mathrm{C}}, \mathrm{p}_{\mathrm{E}}$, and sample size. The resulting values were plotted, and curves were smoothed. In the final graphs $P_{E}$ was represented on the abscissa, power on the ordinate, and sample size on separate power curves within each graph. Levels of $\mathrm{p}_{C}$ were represented by separate graphs.

\section{EVALUATION OF GRAPHICAL AID}

A random sample of 15 articles from the series of negative clinical trials of Freiman et $a l^{6}$ was drawn. For each article cards indicating $\mathrm{p}_{\mathrm{C}}$, sample size ( $\left.\mathrm{n}\right)$, and the $\left|\mathrm{p}_{\mathrm{E}}-\mathrm{p}_{\mathrm{C}}\right|$ which represented a $25 \%$ improvement in response rate were developed. After the evaluation method was piloted and improved, a group of 18 medical students, residents in internal medicine, fellows in medical subspecialties, and faculty was asked to determine the statistical power for each study by using the card abstracts and an early version of the graphical aid. All participants were trained for 15 minutes. The training included discussion of $\alpha, \beta$, sample size, and the need for defining the clinically important difference in order to determine power. During the training period participants were supervised in estimating power for three articles. They then estimated power for the remaining 12 articles without further help. One card was found to be incorrectly designed, and one participant failed to understand the concept and use of the graphical aid. Thus 17 subjects and 11 examples constituted the final evaluation set. The true power was determined for each example using the above formula and a standard $\mathrm{Z}$ table.

Accuracy and reliability were assessed by examining the bias, variance, and mean square error of the power estimates. Bias reflected the accuracy of the graphical aid estimate of the true power. Variance reflected the degree of precision, or reliability, among the users' power estimates. Mean square error was the sum of the variance plus the squared value of the bias.

Estimates of the mean, variance, bias, and mean square error were obtained for each of the 11 examples. For each example bias was estimated by the difference between the average of the users' estimates and the true (formula computed) power. Summary estimates of bias, variance, and mean square error were obtained by taking the average of the 11 estimates.

\section{Results}

\section{USE OF THE GRAPHICAL AID}

The figure displays the graphical aid. To use the aid the user locates the graph that most closely approximates to the success rate in the control group, $\mathrm{p}_{\mathrm{C}}$. If one is evaluating the results of a reported clinical trial $\mathrm{p}_{\mathrm{C}}$ can be estimated by the observed success (failure) rate in the control group and $p_{E}$ is derived from the specified clinically important difference. The user then locates the hypothesised value of $\mathrm{p}_{\mathrm{E}}$ on the abscissa. A vertical line is traced upwards from that point on the abscissa 

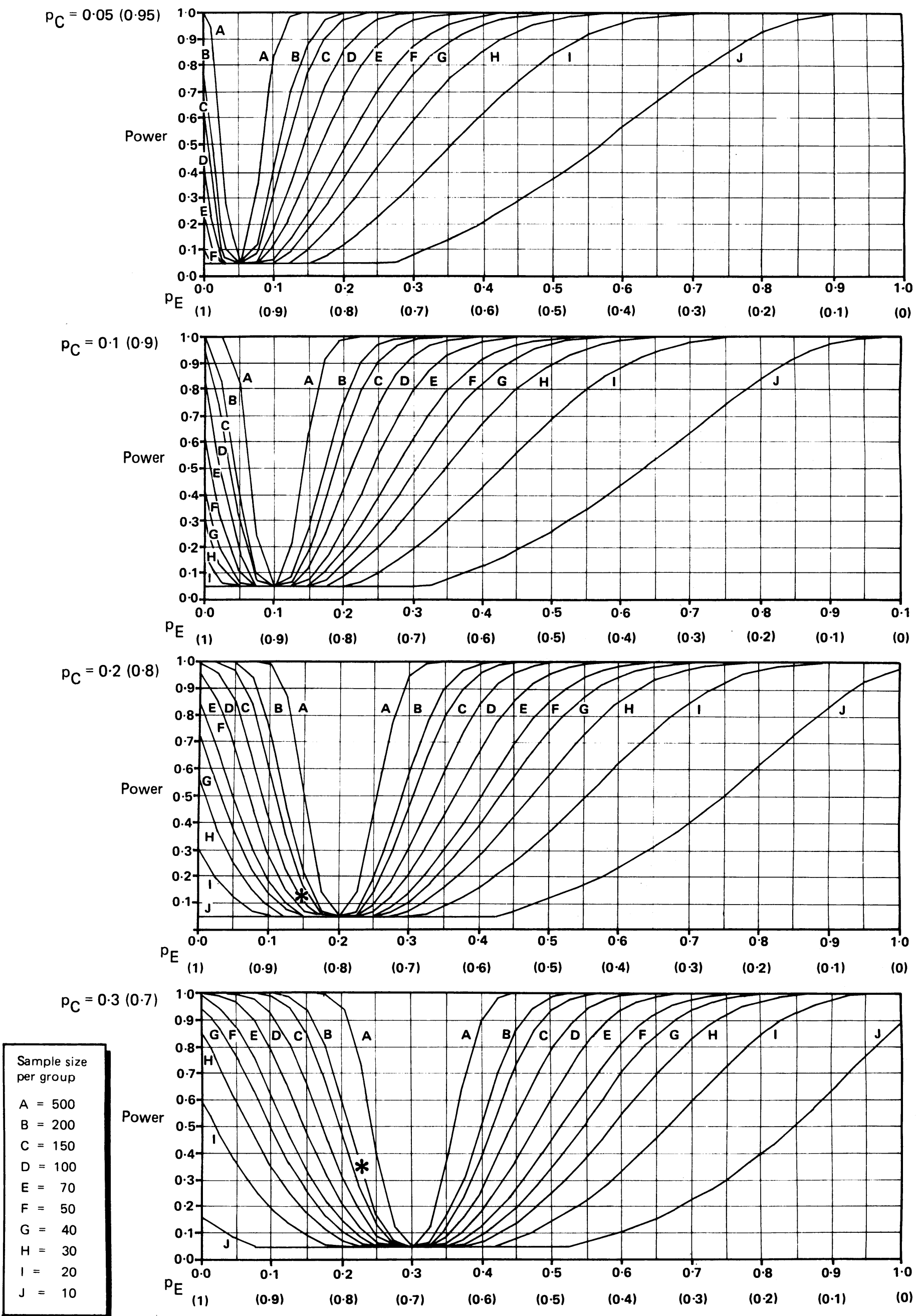


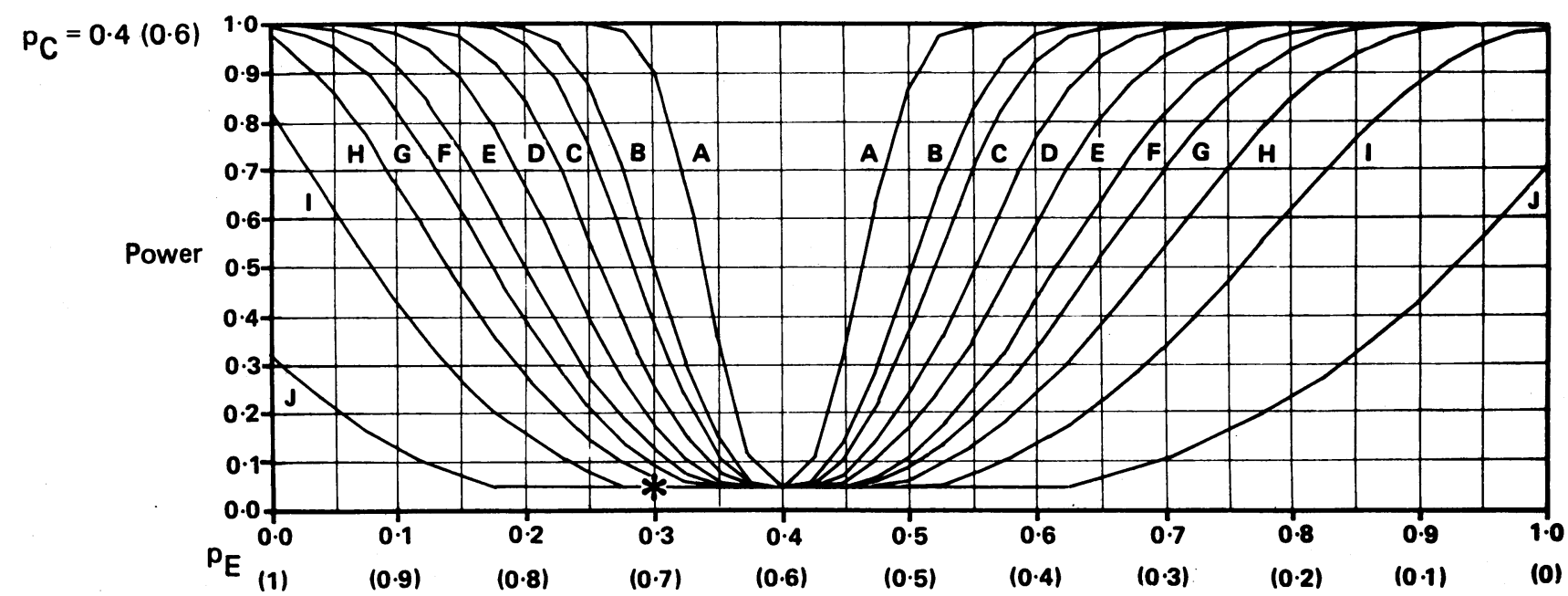

$\begin{aligned} & \text { Sample size } \\ & \text { per group }\end{aligned}$
$A=500$
$B=200$
$C=150$
$D=100$
$E=70$
$F=50$
$G=40$
$H=30$
$I=20$
$J=10$

Sample size

$P_{C}=0.5(0.5)$

1.0

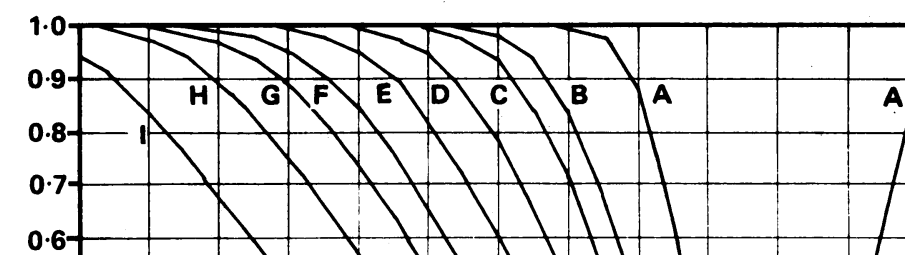

Power
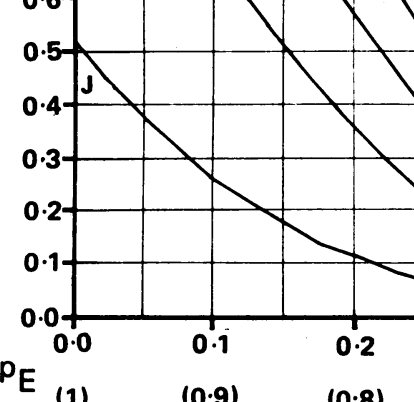

(0.8)

$(0 \cdot 7)$

(0.6)

(0.5)

(0.4)

(0.3)

(0.2)

(0.1)

(0)

Graphical aid for determining the power of clinical trials. Power is represented on the ordinate. The two tailed $\alpha$ significance level is 0.05 . p denotes the hypothesised proportion of respondents in the control group and $p_{E}$ the hypothesised proportion of respondents in the experimental group. Asterisks indicate the graphical coordinates for the three clinical examples (see text)

until it meets the appropriate curve corresponding to sample size. Finally, the user traces a line horizontally to the ordinate level to estimate the power of the study.

The method works best when the sample size $(n)$ is equal in the control and experimental groups and $\mathrm{p}_{\mathrm{C}}$ falls on a graph rather than between graphs. When the two sample sizes are different power will be bounded by the values associated with the smaller and larger sample sizes. Alternatively, the average sample size can be used to obtain a point estimate of power. When $\mathrm{p}_{\mathrm{C}}$ falls between graphs power can be estimated using the graphs immediately above and below the indicated $\mathrm{p}_{\mathrm{C}}$ and the final estimate defined using a linear extrapolation between the two preliminary estimates.

Clinical example 1 -To illustrate we return to the Danish aspirin study discussed by Dyken. ${ }^{3+} \mathrm{p}_{\mathrm{C}}$ is estimated by the observed rate, $0 \cdot 8$, of untreated patients with transient ischaemic attacks who have no disabling stroke or die over two years' follow up. Accepting a $25 \%$ improvement in failure rate to be of practical importance, the research evaluator defines the clinically important difference to be 0.05 -that is, $0.25 \times(1-0.8)=0.05$ and, therefore, $\mathrm{p}_{\mathrm{E}}=0.85\left(\mathrm{p}_{\mathrm{C}}+\right.$ clinically important difference). Because 101 patients received aspirin and 102 placebo, the research evaluator selects the graph representing $\mathrm{p}_{\mathrm{C}}=(0.8), \mathrm{p}_{\mathrm{E}}=(0.85)$, and curve $D$, representing $n=100$, to estimate power to be about $10 \%$. The formula described in the methods section yielded a power of $11 \%$. Because the Danish study had such low power to find a difference of 0.05 the research evaluator cannot conclude from the Danish study that a clinically important difference does not exist.
Clinical example 2-Next consider a double blind randomised trial on the effect of the antiulcer agent sodium amylsulfate on the healing of duodenal ulcer. ${ }^{17}$ Eighteen patients received sodium amylsulfate and 17 a placebo. $p_{C}$ is estimated by the observed rate, 0.59 , of healing in the placebo group. The clinically important difference associated with a $25 \%$ improvement in ulcer healing is 0.1025 and $\mathrm{p}_{\mathrm{E}}=\mathrm{p}_{\mathrm{C}}+$ clinically important difference $=0.6925$. Using the graph for $p_{C}=(0.6)$, $p_{E}=(0 \cdot 7)$, and $n=20$, power is estimated to be $0 \cdot 05$. By formula power was computed as 0.035 .

Clinical example 3-In a randomised controlled trial 184 patients with acute myocardial infarction were treated with recombinant tissue type plasminogen activator, and 183 patients were treated with the same medical regimen plus immediate percutaneous transluminal coronary angioplasty. ${ }^{18}$ Numerous outcomes were reported. The research evaluator decides that the outcome of major interest is the incidence of recurrent coronary ischaemia over the three months after randomisation. $\mathrm{p}_{\mathrm{C}}$ is estimated by the observed rate of 0.30 in the non-invasive strategy group and $\mathrm{p}_{\mathrm{E}}$ is 0 selected as 0.225 , representing a $25 \%$ improvement in the adverse outcome. The evaluator uses the graph for $\mathrm{p}_{\mathrm{C}}=0 \cdot 3$, locates $\mathrm{p}_{\mathrm{E}}$, and estimates power to be $0 \cdot 34$. Computation of power by the formula indicated $0 \cdot 33$. Note, however, that if the evaluator estimates power for several outcomes neither the graphs nor the power formula accurately represents the true power of the experiment.

\section{EVALUATION RESULTS}

Estimated bias ranged between -0.077 and 0.059 
with summary bias equal to -0.0003 . Sample variances ranged between 0.000 and 0.023 with a summary variance of 0.006 . The summary estimate of mean square error was $0 \cdot 007$. Thus, the estimated bias, variance, and mean square error of the power estimates were small. All users correctly determined power within $5 \%$ of the true power in eight or more of the 11 examples, with a $90 \%$ overall rate of correct determinations. Eight of the 17 participants correctly determined power for all examples. After the evaluation the graphs were refined using computer plotting with improved scaling of the abscissa, which should enhance the accuracy of the aid.

\section{Discussion}

Physicians must be able to evaluate clinical research critically. ${ }^{2}$ Determining the power of a study is an essential component of the design and evaluation of clinical research, particularly clinical trials, but power analyses are often not included in published reports. Thus, physicians need simple tools that help them in this aspect of evaluating published reports. The aid presented in this paper can serve this purpose when the outcomes are dichotomous. When the outcomes are continuous power can also be computed through formulas or tables. ${ }^{15}$ Alternatively, one might compare the sample size reported with that required to detect the clinically important difference of interest. For this purpose the simple nomogram provided by Altman would be useful. ${ }^{19}$

One cannot determine power without deciding the difference that it is important to detect. Thus the choice of clinically important difference is a crucial component of power analysis, requires a carefully considered decision, and should be clearly indicated in any description of the analysis. The most useful methods for determining power, including this aid, permit the user to apply indivdual clinical judgment in defining this important variable.

Several caveats in the use of the graphical aid for research evaluation are in order. The aid is not appropriate when the conditions indicated here do not apply. For example, it should not be used when multiple groups are compared or multiple tests are performed. The aid has been limited to a two tailed $\alpha$ of 0.05 because it is the most common convention and is designed for power determination after a study has been completed. When planning trials investigators should consult textbooks ${ }^{1+15}$ or seek advice from a statistician.

The major function of this graphical aid is to enable clinical users to get a quick estimate of the ability of the study to reject a false null hypothesis. In particular, it facilitates the use of individual judgment in the assessment of negative clinical trials. Evaluation of the aid suggests that its use will result in a power estimate within 0.05 of the true power $90 \%$ of the time when the clinical trial in question meets the conditions outlined above. Power tables ${ }^{15}$ or formulas ${ }^{16}$ can be used when the user wants to estimate power with greater accuracy.

Finally, small sample size relates to both low power and greater standard error and thus less precise estimation of both $\mathrm{p}_{\mathrm{C}}$ and $\mathrm{p}_{\mathrm{E}}$. Confidence intervals are particularly valuable for estimating the magnitude of the difference between two groups by providing a range of values for the true difference and the degree of confidence associated with this difference. Confidence intervals thus provide greater information than $P$ values and should be used in reporting the results of clinical trials. ${ }^{2121}$

We thank Ms Penny Jennings, Ms Marylee Reese, and Dr Jeffrey Work for help in computer plotting the power curves and Ms Maxine Lax for her help in preparing the manuscript.
1 Relman AS. A new series on biostatistics. N Engl f Med 1982;306:1360-1. 2 Bennett KJ, Sackett DL, Haynes RB, Neufeld VR, Tugwell P, Roberts R. A controlled trial of teaching critical appraisal of the clinical literature to medical students. FAMA 1987;257:2451-4

3 Sorensen PS, Pedersen H, Marquardsen J, et al. Acetylsalicyclic acid in the prevention of stroke in patients with reversible cerebral ischemic attacks. A Danish cooperative study. Stroke 1983;14:15-22.

4 Dyken ML. Transient ischemic attacks and aspirin, stroke and death; negative studies and Type II error. Stroke 1983;14:2-4.

5 Browner WS, Newman TB. Are all significant $P$ values created equal? The analogy between diagnostic tests and clinical research. JAMA 1987;287: 2459-63.

6 Freiman JA, Chalmers TC, Smith H, Kuebler RR. The importance of beta, the type II error and sample size in the design and interpretation of the randomized control trial. N Engl F Med 1978;299:690-4.

7 Reed JR III, Slaichert W. Statistical proof in inconclusive 'negative' trials. Arch Intern Med 1981;141:1307-10.

8 Lachin JM. Introduction to sample size determination and power analysis for clinical trials. Controlled Clinical Trials 1981;2:93-113.

9 DerSimonian R, Charrett LJ, McPeek B, Mosteller F. Reporting on methods in clinical trials. N Engl Y Med 1982;306:1332-7.
ing

10 Emerson JD, Colditz GA. Use of statistical analysis in the New England Journal of Medicine. N Engl f Med 1983;309:709-13.

11 Feigl P. A graphical aid for determining sample size when comparing two independent proportions. Biometrics 1978;34:111-22.

12 Young MJ, Bresnitz EA, Strom BL. Sample size nomograms for interpreting negative clinical studies. Ann Intern Med 1983;99:248-51.

13 Detsky AS, Sackett DL. When was a 'negative' clinical trial big enough? How many patients you needed depends on what you found. Arch Intern Med 1985;145:709-12.

14 Fleiss JL. Statistical methods for rates and proportions. 2nd ed. New York: John Wiley, 1981.

15 Cohen J. Statistical power analysis for the behavioral sciences. 2nd ed. New York: Academic Press, 1977.

16 Fleiss JL, Tytun A, Ury HK. A simple approximation for calculating sample sizes for comparing independent proportions. Biometrics 1980;36:343-6.

17 Landeker KD, McCallum EM, Fevre DI, Green PH, Kasunle A, Piper DW. Effect of sodium amylsulfate (Depepsen) on the healing of duodenal ulcer Gastroenterology 1976;71:723-5.

18 Simoons ML, Arnold AER, Betriu A, et al. Thrombolysis with tissue plasminogen activator in acute myocardial infarction: No additional benefit from immediate percutaneous coronary angioplasty. Lancet 1988;i:197-202. 19 Altman DG. Statistics and ethics in medical research, III. How large is a sample? Br Med f 1980;281:1336-8. (Reprinted in Gore SM, Altman DG. Statistics in practice. London: BMA, 1982).

20 Gardner MJ, Altman DG. Confidence intervals rather than $P$ values: estimation rather than hypothesis testing. Br Med f 1986;292:746-50.

21 Simon R. Confidence intervals for reporting results of clinical trials. Ann Intern Med 1986;105:429-35.

(Accepted 25 May 1988)

\section{ANY QUESTIONS}

Is there any reason why a healthy person who has had a splenectomy should not travel abroad?

Immediately after splenectomy haemostatic and thromboembolic complications may occur and travel should not normally be undertaken until these risks are pastusually in four to six weeks. In the longer term patients who have had a splenectomy have an increased risk of serious infections including septicaemia, and so travellers should be warned of any particular infection risks in the countries concerned, take precautions in their lifestyle, and have the appropriate immunisations. Malaria prophylaxis is especially important, and pneumococcal and meningococcal vaccines should be considered. Knowledge of reliable local medical facilities may usefully be obtained in advance of any illness, particularly by longer stay travellers.-ERIC WALKER, lecturer in infectious diseases, Glasgow.

\section{Correction}

\section{The eye and the nervous system}

We regret that an editorial error occurred in this $\mathrm{ABC}$ of Eyes by Messrs A R Elkington and P T Khaw (2 July, p 59). Infected was substituted for injected in the first sentence of the section on the red eye under the heading "Headaches and the eye" (p 62). The correct sentence should read, "A red eye-In acute glaucoma the eye is usually red, injected, and tender, and the acuity is diminished."

\section{General medical disorders and the eye}

An authors' error occurred in this $A B C$ of Eyes by Messrs A R Elkington and P T Khaw (6 August, p 412). On page 415 under "Rheumatoid arthritis" the pictures of episcleritis and scleritis were transposed. 